\title{
Visibility Enhancement and Power Saving by Pro-beam LED Tunnel Lighting Method
}

\author{
Motohisa SATO and Tomokazu HAGIO
}

NEXCO Expressway Research Institute of Japan

Received July 25, 2013, Accepted September 30, 2013

\begin{abstract}
The symmetrical lighting system, which is appropriate to recognize an object with silhouette vision, has been used for many years as tunnel interior lighting. The pro-beam lighting system however has an advantage to enhance visibility with reversed silhouette and has being investigated for more safe and comfortable driving in the tunnel for several years by the NEXCO group, especially to increase visibility for a preceding vehicle to prevent rear-end collisions. The pro-beam lighting with ceramic metal halide lamps was largely adapted for 70 tunnels of New Tokyo-Nagoya expressway in 2012, and further study has being made to achieve both good visibility and power efficiency with the use of LED pro-beam lighting. In this paper, the research concerning to pro-beam lighting are described such as visibility analysis of LED tunnel lighting, enhanced visibility by pro-beam, and evaluation of experimental installation by both field observations and the mathematical values of total revealing power. The evaluation of the LED pro-beam tunnel showed good visibility and power efficiency.
\end{abstract}

KEYWORDS: tunnel interior lighting, LED, pro-beam lighting, uniformity, total revealing power

\section{Introduction}

The symmetrical lighting system has been used for many years as tunnel interior lighting, and this method is appropriate to see a dark object which has a small reflectance factor with silhouette vision, but not so effective in terms of seeing a bright object which has a high reflectance factor or seeing a preceding vehicle. We examined the causes of tunnel accidents and found there were fewer accidents due to fallen objects, but were more rear-end collisions. This means if you could increase visibility of preceding vehicles you could drive more safely. Pro-beam lighting is one of the best solutions to increase visibility of preceding vehicles since the tunnel interior is lighted forward you drive and you can see the rear of the preceding vehicles better.

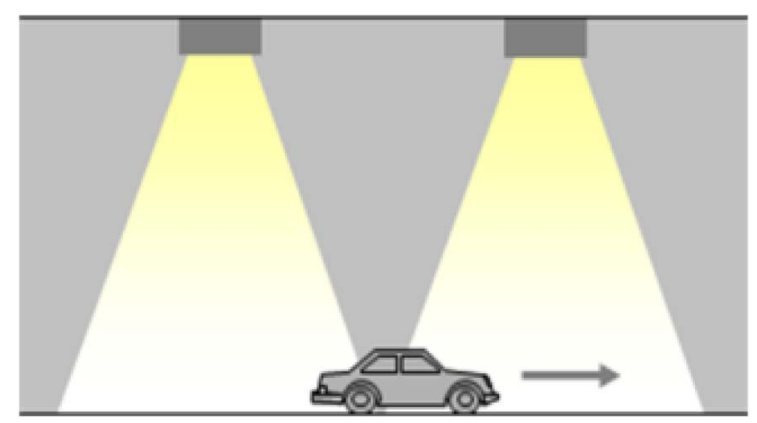

Figure 1 Symmetrical lighting

\section{Conventional tunnel lighting}

The symmetrical lighting system by fluorescent or sodium lamps has been used for expressway tunnels with the number of more than 1,600 in Japan. The lighting fixtures are installed along both sides of the tunnel ceiling to make maintenance work easier.

Under the symmetrical system, it is good to see objects with low reflectance factors because objects with less than $20 \%$ reflectance factors were accounted for $90 \%{ }^{1)}$ and good to be seen by silhouette vision.

3. High overall luminance uniformity leads to reduction of average road luminance and power consumption

The overall luminance uniformity of road surface in a tunnel is designed with the value of 0.4 or more as CIE recommendation. If the value is increased significantly, can the average road luminance be lowered? Figure 2 shows the comparison between two tunnels with typical uniformity and very high one. LED has advantages in flexible design of luminaries, so the compact luminaries are used and the better uniformity is achieved since the spacing of luminaries can be much closer. Even with the same value of average luminance, you can see clear difference in visibility between them. According to the study by Narisada ${ }^{2}$, he found the relation of necessary overall uniformity and average road surface luminance with the parameters of contrast value (Figure 


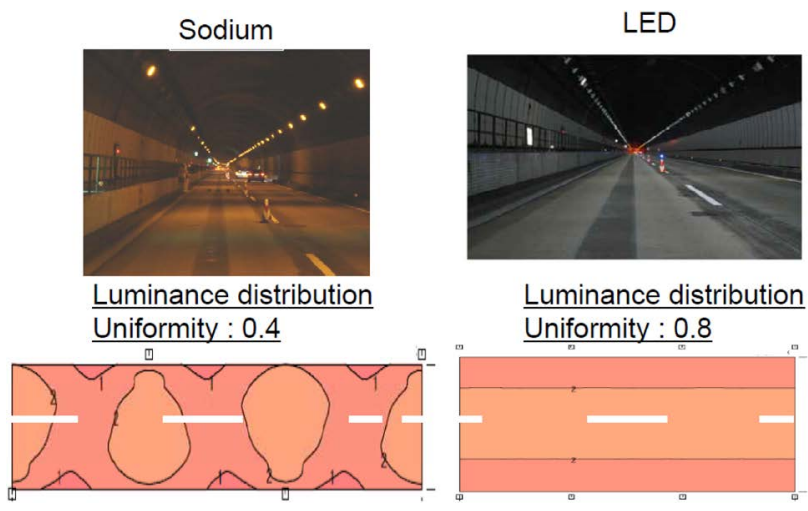

Figure 2 Comparison between different uniformity and lamp

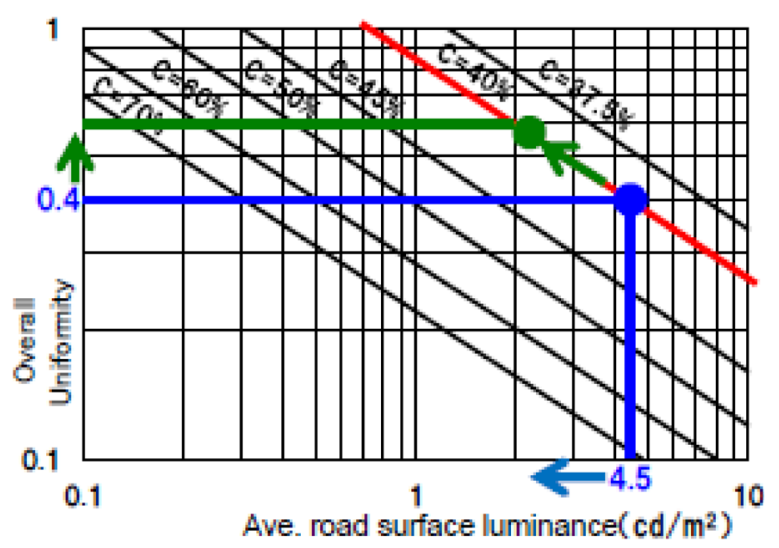

Figure 3 Relationship between necessary uniformity and average road surface luminance

3). He made the indoor experiment to find the probability of perception under various visual environments with the variables of average road luminance $\mathrm{L}$ and overall luminance uniformity $U$, and then found Figure 3 which shows the inverse proportion between $\mathrm{U}$ and $\mathrm{L}$ as a parameter of contrast between fallen objects and road surface. This means that higher $\mathrm{L}$ is required when $U$ is low (bad), but the same visibility can be obtained even with lower value of $\mathrm{L}$ when $\mathrm{U}$ is high (good) under some constant sight distance. For example, if you increase the overall uniformity such as from 0.4 to 0.6 , the average luminance could be lowered to nearly $2 \mathrm{~cd} / \mathrm{m}^{3}$ as shown in Figure 3, meaning that you could decrease the average luminance when the uniformity were raised, so the power could be decreased.

\section{Contribution to safety-increasing visibility by pro-beam}

According to the NEXCO traffic accident data in expressway tunnels from 2006 to 2008, 24\% of accidents were caused by rear-end collisions while $18 \%$ were by fallen objects. The tunnel lighting has been designed how small fallen objects, $20 \times 20$ centimeters can be visible with silhouette vision on the supposition that most
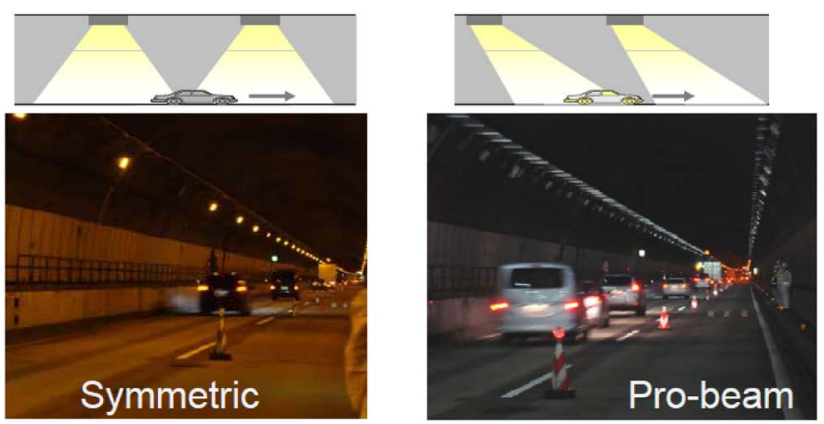

Figure 4 Difference in looking of the rear of preceding vehicles between symmetric and pro-beam lighting

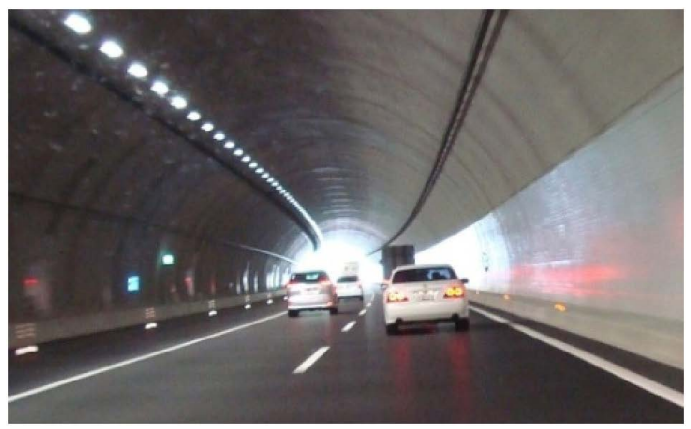

Figure 5 Pro-beam tunnel lighting in Shin Tomei Expressway

objects are with small reflectance factors $(90 \%$ of objects were less than 20\% reflectance by Smith: see Figure 6), but the visibility of preceding vehicles is important for the safety in tunnels considering the ratio of rear-end collision. Figure 4 shows how the visibility of the rear of preceding vehicles is different under the lighting environment of symmetric and pro-beam.

Pro-beam lighting is expected to be very effective especially for tunnels with high traffic volumes since you have more possibility to drive in tunnels following preceding vehicles. The $160 \mathrm{~km}$ section of the ShinTomei Expressway between Tokyo and Nagoya was opened in April 2012 and pro-beam lighting with ceramic metal halide lamps was adapted for all 70 tunnels since large traffic volumes are expected (Figure 5).

\section{Distribution of reflectance factors of fallen objects and their visibility}

As it is referred in Chapter 2 and 4, objects with low reflectance factors, less than $20 \%$, were supposed to be accounted for 90\% shown in Smith Study line in Figure 6. So, tunnel lighting has been designed to recognize dark objects with silhouette vision. The data relating to reflectance factors of actual fallen objects on the expressways was studied and analyzed recently in Ja$\mathrm{pan}^{3)}$. The ratio of dark objects was much less in this study (Our Research line in Figure 6), and it means 35\% 


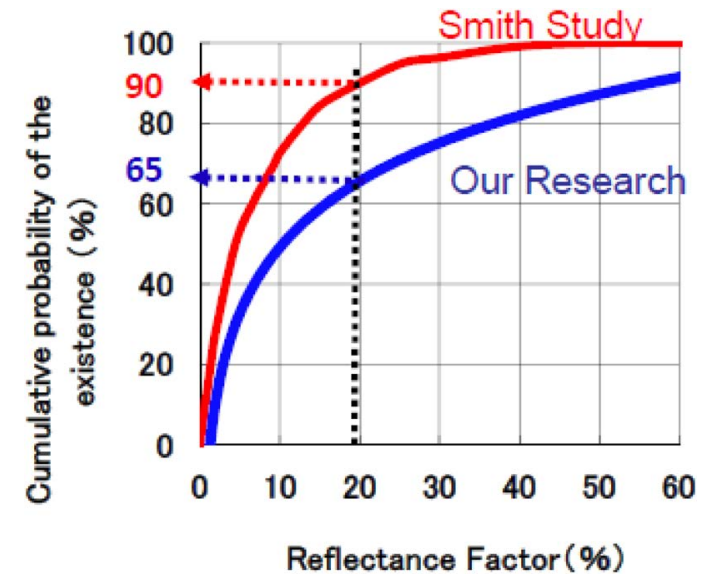

Figure 6 Cumulative probability of the existence to reflectance factors of fallen objects

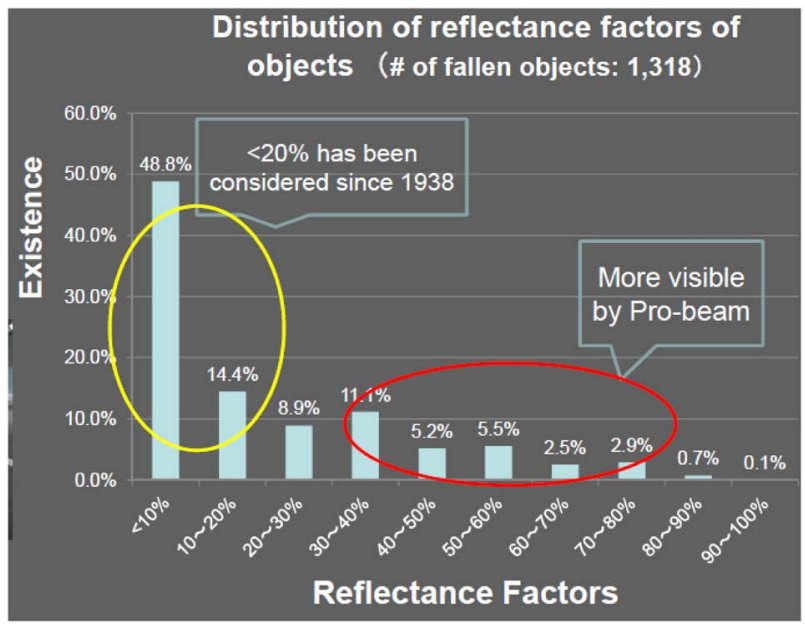

Figure 7 Distribution of reflectance factors of fallen objects

of fallen objects with higher than $20 \%$ reflectance may not be well visible under the conventional lighting as shown in Figure 7.

\section{Experimental installation of LED pro-beam light- ing}

The LED pro-beam lighting has been installed in two tunnels to do research on visibility to find how objects with higher reflectance factors are recognized and how preceding vehicles are seen well under the pro-beam lighting, and also how visibility varies when pro-beam lighting is dimmed by $50 \%$. The length of one tunnel is $2314 \mathrm{~m}$ with one way two lanes. The new LED probeam luminaries were installed on each side of upper sides with the space of $3.9 \mathrm{~m}$ while the existing conventional low pressure sodium lamp luminaries with symmetric system were stayed there also for visibility study. The average road luminance of $100 \%$ pro-beam, $50 \%$ pro-beam, and symmetrical lighting was $3.1 \mathrm{~cd} / \mathrm{m}^{3}$, $1.6 \mathrm{~cd} / \mathrm{m}^{3}$, and $3.6 \mathrm{~cd} / \mathrm{m}^{3}$ respectively. The pictures of

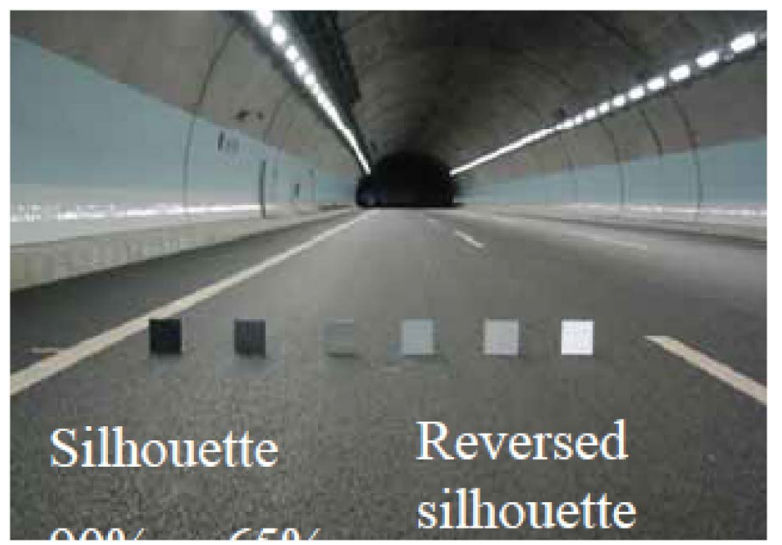

Figure 8 Sample objects with various reflectance factors

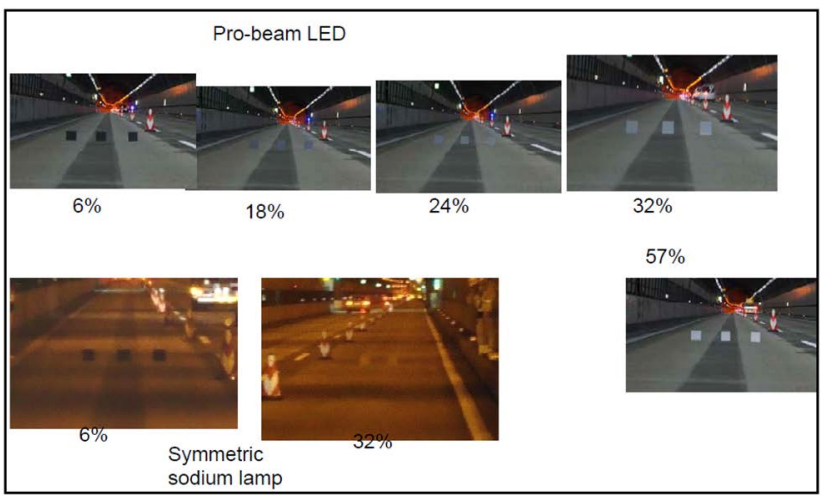

Figure 9 How objects with different reflectance factors look under pro-beam and symmetrical lighting

Table 1 Scales for observers

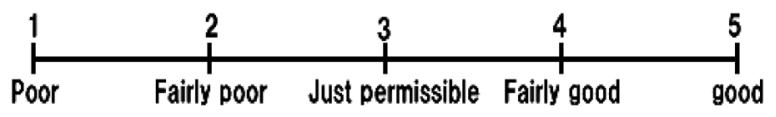

the objects with various reflectance factors are shown in Figure 8. It is clear that objects with higher reflectance factors can be well visible in the LED pro-beam than the sodium symmetric.

\section{Result of observations}

Two kinds of observations were conducted in the tunnel by 23 males aged from 19 to 64 for pro-beam lighting and 8 males from 28 to 49 for symmetrical lighting. Firstly, observation of a vehicle placed in front was conducted, by observers seated in a car to observe the vehicle with grey color which represents, a preceding vehicle, located $80 \mathrm{~m}$ ahead of them. The vehicle was placed where the vertical illuminance was lowest and visibility would be lowest. For example, since the road surface luminance is uneven due to low value of uniformity in case of the sodium symmetric, the vehicle was placed right under a luminaire where the illuminance of 


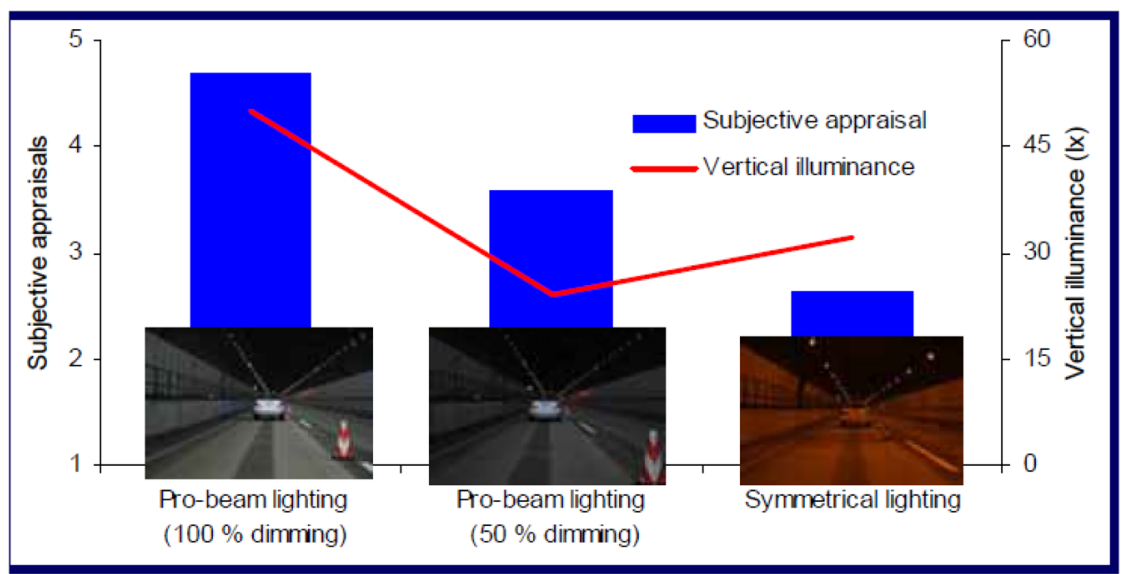

Figure 10 Subjective appraisals of a vehicle located ahead

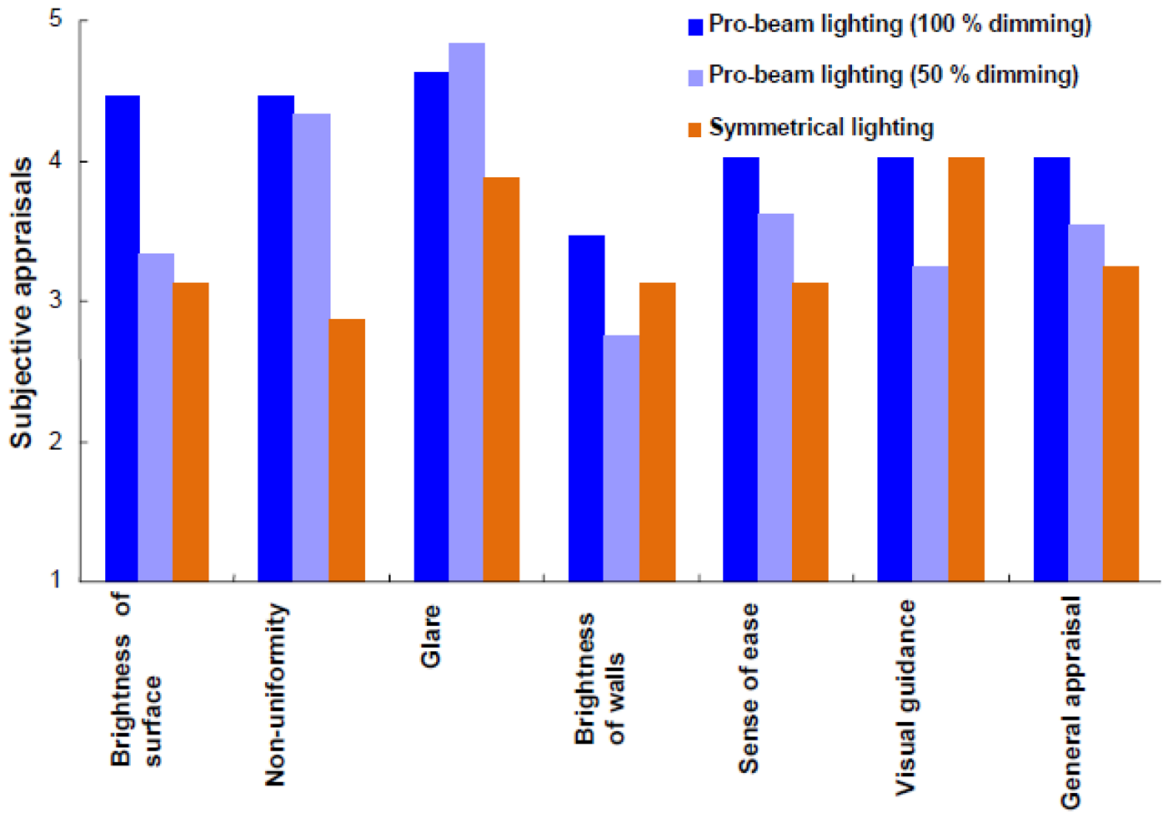

Figure 11 Subjective appraisals of visual environment

the vehicle's rear is at the lowest although the road surface luminance is highest. The observers were asked to appraise subjectively on a scale of 5 in Table 1 .

In the pro-beam lighting, the subjective appraisal in condition of $50 \%$ dimming is lower than that in condition of full output, but gotten a little over 3, "Just permissible" or more, while vertical illuminance of $50 \%$ dimming of pro-beam lighting is lower than that of the symmetrical lighting system (Figure 10) ${ }^{4}$. This result shows a preceding vehicle is well visible in the condition of pro-beam even when vertical illuminance is relatively low.

Secondly, observers were asked to appraise subjectively seven factors for visual environment on a scale of 5. Subjective appraisal of the 50\% dimming pro-beam was mostly good except for brightness of the wall and visual guidance. The study of subjective appraisals of visual environment showed that the pro-beam lighting with certain road luminance faired very well in all aspects, and when the luminance was halved, it remained still fair. However, the appraisal points of the symmetrical lighting were not high in spite of higher road luminance to that of the pro-beam (Figure 11$)^{4}$.

\section{Evaluation of visibility by total revealing power}

TRP (Total Revealing Power) is defined as the sum of the revealing power for the silhouette vision and the reversed silhouette vision. As shown in Figure 123), the revealing power of silhouette vision decreases as the vertical illuminance goes up, but the one of reversed silhouette increases as the vertical illuminance goes down. However, TRP stays constant from the certain value of illuminance.

The value of TRP in the experimental tunnel was 


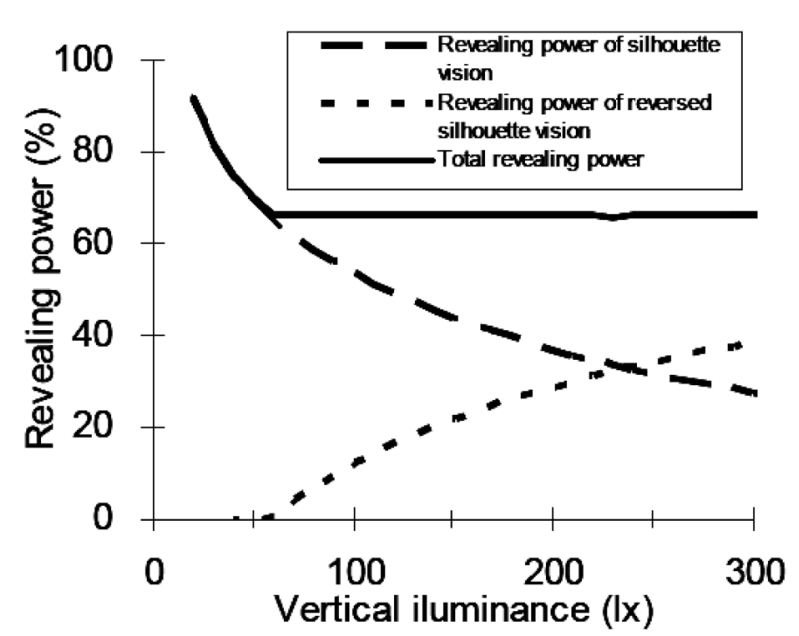

Figure 12 Relationship between revealing power and vertical illuminance

found to be $86.6 \%, 83.3 \%$, and $87 \%$ for $100 \%$ pro-beam, $50 \%$ pro-beam, and symmetrical lighting respectively. The visibility of two lighting systems did not have remarkable differences between the values of TRP, which means that the visibility does not decline so much even when the average road surface luminance is reduced to the half-from 3.1 to $1.6 \mathrm{~cd} / \mathrm{m}^{3}$.

Although the pro-beam was rated better than the symmetric in the subjective appraisals, the values of TRP were not higher than the symmetric. It is because TRP is an index to represent the visibility of fallen objects. The values of TRP tend to be higher for symmetrical lighting which is advantageous in silhouette vision because the majority of fallen objects are with low reflectance factors.

However, it is important to consider the overall visual environment in a tunnel for safe and comfortable driving, not only fallen objects but also preceding vehicles, the walls, or the lane markings on the road surface. The pro-beam lighting has an advantage especially in tunnels where traffic volumes are large as you have more chances to follow vehicles in driving rather than just watching fallen objects ahead.

There will be another reason of less difference in TRP. The gap of the critical reflectance factors between silhouette and reversed silhouette vision was rather large in the experimentally installed tunnel. If the lighting design such as angle of optical axis of the luminaries is optimized to minimize the gap, the pro-beam should perform better. This is the subject for our further study.

\section{Conclusion}

We have analyzed the visibility of the preceding vehicles and the visual environment in the tunnel based on the experimental installation of the LED pro-beam lighting.

(1) The visibility of the preceding vehicles by the probeam lighting was much better compared with the one by the symmetrical lighting even when the road surface luminance was lower than the symmetrical system and was reduced by half.

(2) The study of subjective appraisals of visual environment showed that the pro-beam lighting with certain road luminance was rated very well in all aspects, and when the luminance was halved, it remained still fair. However, the appraisal points of the symmetrical lighting were not high in spite of higher road luminance comparing to that of the probeam.

(3) There was not much difference between the probeam lighting and symmetrical lighting in the overall visibility of fallen objects in terms of TRP in spite of different average road surface luminance.

(4) By adopting the pro-beam lighting system, the average road surface luminance can be reduced and power saving can be made.

\section{References}

(1) Smith, F.: Reflection factors and revealing power, Trans. Illum. Engng. Soc. (London), 3, 196206. pp. 196-206 (1938).

(2) Narisada, K.: Influence of non-uniformity in road surface luminance of public lighting installations upon perception of objects on the road surface by car-drivers, Proceedings of the 17th Session of CIE, (1971).

(3) Hirakawa, S. et al: Total revealing power for tunnel lighting (second report), Proceedings of the 26th the Session of the CIE, D4 pp. 148-151 (2007).

(4) Ito, H. et al: Visibility of the critical object and energy efficiency of pro-beam lighting for tunnel interior lighting, Proceedings of the CIE Midterm Conference (Paris) , D4 pp. 1083-1091 (2013). 